\title{
Results from the Pierre Auger Observatory
}

\author{
G. Rodriguez ${ }^{1}$ for the Pierre Auger Collaboration ${ }^{2,3}$
}

${ }^{1}$ Universidad de Santiago de Compostela, Facultad de física, Spain, Email: grod@fpaxp1.usc.es

2 Av. San Martín Norte 304 (5613) Malargüe, Prov. Mendoza, Argentina

${ }^{3}$ A full author list and affiliations can be found at http://www.auger.org/archive/authors_2010_10.html

DOI: http://dx.doi.org/10.5689/UA-PROC-2010-09/30

\begin{abstract}
Ultra-high energy cosmic rays are observed at the Pierre Auger Observatory, the largest cosmic ray experiment in operation, through a hybrid technique employing fluorescence and surface detectors. These observations provide results on the energy and arrival directions of the highest energy cosmic rays, and also they allow to characterize the extensive air showers they induce, probing the nature of the primary particles and their interactions.
\end{abstract}

\section{INTRODUCTION}

The cosmic ray energy spectrum extends to extremely high energies, up to at least $10^{20} \mathrm{eV}[1]$. Due to the steepness of the spectrum, these ultra-high energy cosmic rays (UHECRs) cannot be detected directly, but can be studied by detection of the extensive air showers produced when they interact in Earth's atmosphere. This allows construction of large arrays which provide the collection area necessary to detect such rare particles, arriving with a flux of 1 per $\mathrm{km}^{2}$ per century.

The study of UHECRs provides a number of possibilities to probe physics beyond the Standard Model. While the conventional explanation for the source of UHECRs is astrophysical accelerators, the possibility exists that they instead come from the decay of super-heavy particles created in the early Universe. These so-called "top-down" models can be directly tested, as they also predict a sizable high-energy photon and/or neutrino component of the UHECR flux.

\section{THE PIERRE AUGER OBSERVATORY}

The Pierre Auger Observatory [2] is a hybrid cosmic ray air shower experiment that uses different techniques to detect extensive air showers and reconstruct their energy, direction, and particle type. The southern site of the observatory, located in Mendoza, Argentina, was completed in 2008 and covers an area of $3000 \mathrm{~km}^{2}$ (see Fig. 1).

An array of Cherenkov detectors on the ground sample the particle densities as the air shower arrives at the Earth's surface. The Surface Detector (SD) consists of 1600 water-Cherenkov stations arranged on triangular grid with $1.5 \mathrm{~km}$ spacing. The SD has a $100 \%$ duty cycle, and the trigger efficiency for events with zenith angles less than $60^{\circ}$ is approximately $100 \%$ for proton and iron primaries with energies above $3 \times 10^{18} \mathrm{eV}$ [3]. The timing of the signals in the stations is used to reconstruct the arrival direction, typically to better than $1^{\circ}[4]$. 
The longitudinal shower development is observed by the Fluorescence Detector (FD), consisting of 24 telescopes that overlook the SD at four sites around the array [5]. Each telescope uses a mirror and an array of 440 photomultiplier tubes to track the fluorescence light emitted by excited nitrogen molecules as the air shower deposits energy into the atmosphere. Integrating this light deposition provides a nearly calorimetric measurement of the shower energy. The duty cycle of the FD is approximately $10 \%$, as its use requires moonless and dark nights. The hybrid events, which are air showers detected by both instruments, are very precisely measured and provide the energy calibration tool. Hybrid events makes possible to relate the shower energy measured by the FD to the ground parameter energy estimator.

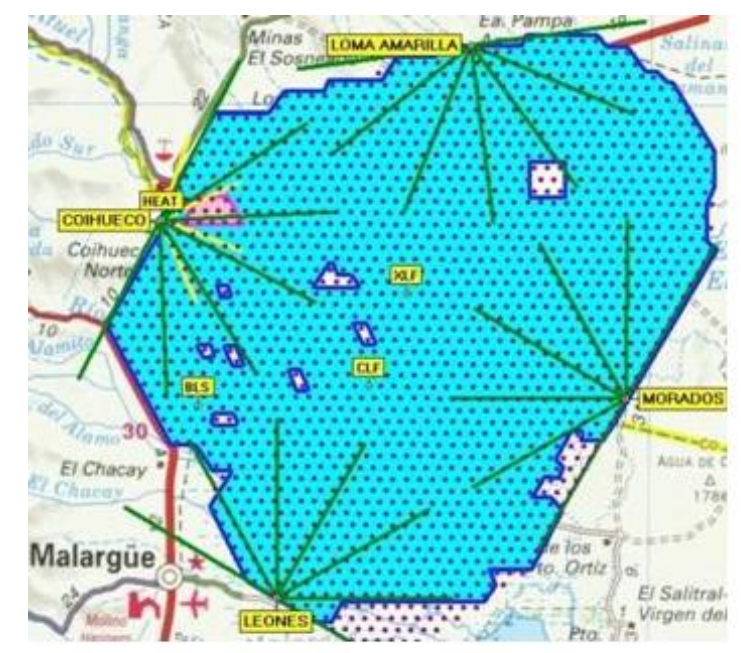

Figure 1: Layout of the southern site of the Pierre Auger Observatory. The red points indicate the Surface Detector stations, and the green rays indicate the field of view of each of the 24 telescopes of the Fluorescence Detector.

\section{RESULTS}

\subsection{Energy Spectrum}

The characteristic features of the combined spectrum [6] are quantified in two ways. For the first method, shown as a dotted red line in Fig. 2, we have used three power laws with free breaks between them. A continuation of the power law above the ankle to highest energies can be rejected with more than $20 \sigma$. For the second characterisation we have adopted two power laws in the ankle region and a smoothly changing function at higher energies, it is showed in black line in the same figure. The position of the ankle has been determined from the fit at $\log _{10}\left(E_{\text {ankle }} / \mathrm{eV}\right)=18.61 \pm 0.01$.

Both the HiRes and Auger experiments have observed a suppression in the spectrum above $3 \times 10^{19} \mathrm{eV}$ [7]. This suppresion can be due to the GZK effect, however, other explanations of the spectral steepening, such as a cutoff in the source spectrum, cannot be ruled out. 


\subsection{Composition}

The atmospheric depth, $X_{\max }$, at which the longitudinal development of a shower reaches its maximum in terms of the number of secondary particles is correlated with the mass of the incident cosmic ray particle, as showers from protons, heavy nuclei, photons, and neutrinos will interact at different depths in the atmosphere and will develop differently. At a given energy, showers from iron primaries will interact higher in the atmosphere and deposit their energy sooner than proton showers. This penetration depth is characterized by $X_{\max }$, the integrated density in $\mathrm{g} / \mathrm{cm}^{2}$ of the shower maximum in the atmosphere. Fluctuations in $X_{\max }$ from shower to shower will be smaller, as to first order an iron shower can be considered as a superposition of single-nucleon showers. The uncertainty in reconstruction of $X_{\max }$ as determined with events recorded at multiple FD sites is $20 \mathrm{~g} / \mathrm{cm}^{2}$ [8] .

Shower $\left\langle X_{\max }>\right.$ and fluctuations $\operatorname{rms}\left(X_{\max }\right)$ as a function of energy are shown in Fig. 3 , measured using nearly 4000 events above $10^{18} \mathrm{eV}$. Both measurements suggest that the composition becomes heavier above $3 \times 10^{18} \mathrm{eV}$, if the hadronic interaction models are correct at these energies. However, an increase in the proton cross section may also explain some of these features [9].

\subsection{Upper limits to photon Fraction}

Photons are expected to dominate over nucleon primaries in non-acceleration models of UHE cosmic-ray origin. Numerous models exist for such "top-down" scenarios, such as super-heavy dark matter (SHDM), topological defects (TD), and interactions with the relic neutrino background (Z-bursts), but many share the common feature of predicting a significant fraction of photons in the UHECR flux from $\sim 10 \%$ at $10^{19} \mathrm{eV}$ to over $50 \%$ at $10^{20} \mathrm{eV}$.

Photon-initiated showers penetrate more deeply and have a higher $X_{\max }$ than protoninduced showers at a given energy, due to decreased secondary multiplicities and suppression of cross sections by the LPM effect. Events with unexpectedly large $X_{\max }$ can be searched for directly with the FD, or shower parameters such as the radius of curvature and signal risetime can be used with the SD. The techniques are effective in different energy ranges, with the FD search effective at EeV energies and the SD at energies above $10 \mathrm{EeV}$. In both analyses, data are consistent with only proton or nuclear primaries [10, 11]. Upper limits on the photon fraction are shown in left panel of Fig. 4 and strongly constrain many top-down models of UHECR production.

\subsection{Upper limits to neutrinos Flux}

The SD of Auger can also be used as a neutrino detector, by searching for highly inclined showers near the horizon. Only a neutrino primary can penetrate deep into the atmosphere before interacting near the detector, so discriminating between "old" versus "young" showers can reject the background of conventional cosmic rays. In practice, this is achieved by using the width of the time distribution of the signals recorded in the Cherenkov stations: "old" showers consisting primarily of a muon bundle will result in a sharp, narrow signal compared to "young" showers with a large electromagnetic component. In addition, $\nu_{\tau}$ can skim through a chord of the Earth, regenerating via multiple $\tau$ production and decay cycles and resulting in an "up-going" shower at the array [12].

No candidate neutrino events have been observed, resulting in upper limits on the diffuse 
flux of neutrinos, see right Fig. 4. The upper limit at the $90 \%$ confidence level on a diffuse $\mathrm{E}^{-2}$ $\nu_{\tau}$ flux is $E^{2} d N / d E<1.3 \times 10^{-7} \mathrm{GeV} \mathrm{cm}^{-2} \mathrm{~s}^{-1} \mathrm{sr}^{-1}$.

\subsection{Arrival direction distribution}

Cosmic rays, being primarily charged particles, are deflected by galactic and extragalactic magnetic fields, and so directional information about their sources is lost. However, for protons primaries above 50-60 EeV, the deflection may only be a few degrees, allowing correlations with sources classes of objects.

In 2007, an a priori analysis provided evidence of angular correlation of events above 57 $\mathrm{EeV}$ with a subset of nearby active galatic nuclei (AGN), with $z<0.018$ and angular separation of $3.1^{\circ}$, from the Véron-Cetty catalog [4]. A follow-up analysis [13] includes an additional 31 high-energy events recorded since the initial publication. The amount of correlation observed it was $69 \%$ in the early data and its current estimate is $38 \%$, while the isotropic expectation is $21 \%$ . A posteriori investigations into clustering in the region around Centaurus A and correlations with other catalogs, such as SWIFT-BAT, are in progress[14].

\section{Acknowledgments}

We thank Ministerio de Ciencia e Innovación (FPA 2007-65114 and Consolider CPAN); Xunta de Galicia (PGIDIT 06 PXIB 206184 PR) and Conseller' ia de Educación (Grupos de Referencia Competitivos é Consolider Xunta de Galicia 2006/51); and Feder Funds, Spain. We also thank CESGA (Centro de Supercomputacion de Galicia) for computing resources.

\section{Bibliography}

\section{References}

[1] M. Nagano, A. A. Watson. Rev. Mod. Phys. 72 (2000) 689.

[2] J. Abraham et al. [Pierre Auger Collaboration], Nucl. Instr. Meth. Phys. Res. A 523 (2004) 50

[3] J. Abraham et al. [Pierre Auger Collaboration], Nucl. Instr. Meth. Phys. Res. A 613 (2010) 29

[4] J. Abraham et al. [Pierre Auger Collaboration], Science 318 (2007) 939

[5] J. Abraham et al. [Pierre Auger Collaboration], Nucl. Instr. Meth. Phys. Res. A 620 (2010) 227.

[6] J. Abraham et al. [Pierre Auger Collaboration], Phys. Lett. B 685 (2010) 239

[7] R. U. Abbasi et. al. Phys. Rev. Lett. 100 (2008) 101101

[8] J. Abraham et al. [Pierre Auger Collaboration], Phys. Rev. Lett. 104 (2010) 091101

[9] R. Ulrich et al. Nucl. Phys. Proc. Suppl. B 196 (2009) 335.

[10] J. Abraham et al. [Pierre Auger Collaboration], Astropart. Phys. 31 (2009) 399.

[11] J. Abraham et al. [Pierre Auger Collaboration], Astropart. Phys. 29 (2008) 243.

[12] J. Abraham et al. [Pierre Auger Collaboration], Phys. Rev. D 79 (2009) 102001.

[13] J. Abreu et al. [Pierre Auger Collaboration], Astropart. Phys. 34 (2010) 314.

[14] J. D. Hague [Pierre Auger Collaboration], Proc. 31st Intl. Cosmic Ray Conf. (Lódź,Poland) [arXiv:0906.2347[astro-ph]]. 


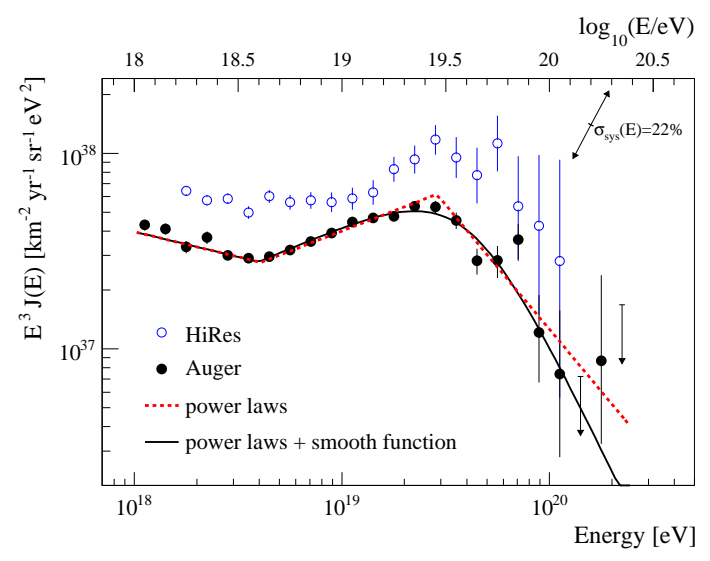

Figure 2: Combined SD and FD Energy Spectrum (multiplied by $E^{3}$ ). Data points from HiRes experiment (blue points) are show for comparison
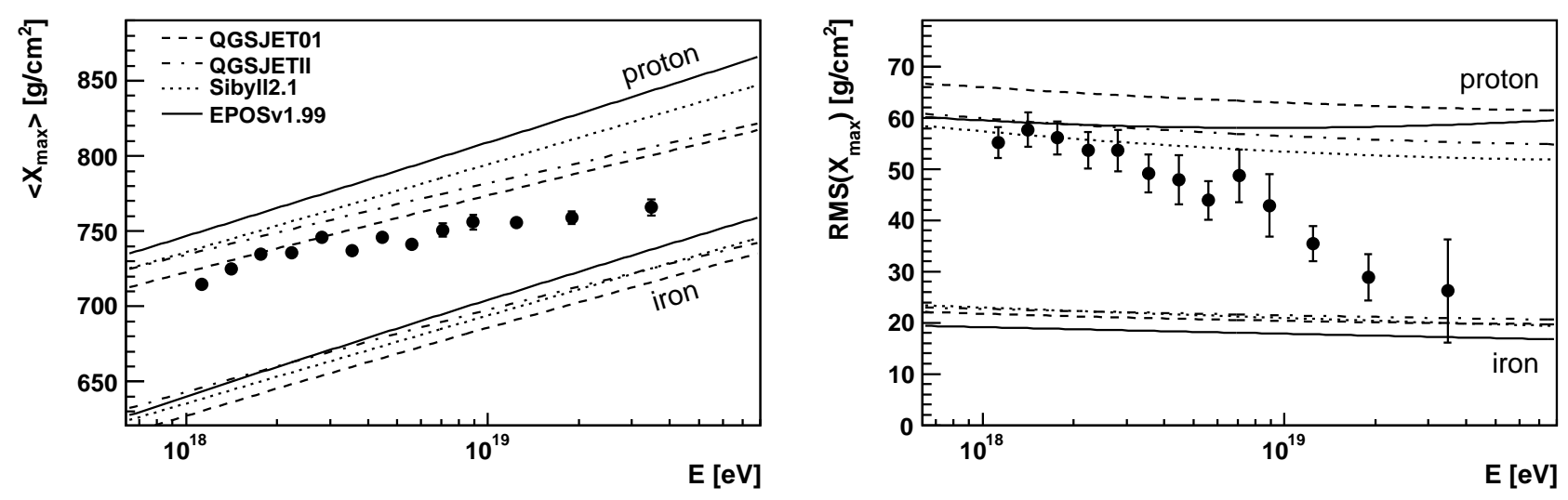

Figure 3: Left panel $\left\langle X_{\max }\right\rangle$ as a function of the energy. Right panel $\operatorname{RMS}\left(X_{\max }\right)$ as a function of the energy. Simulations of proton and iron primaries using various hadronic interaction models are shown as various lines 

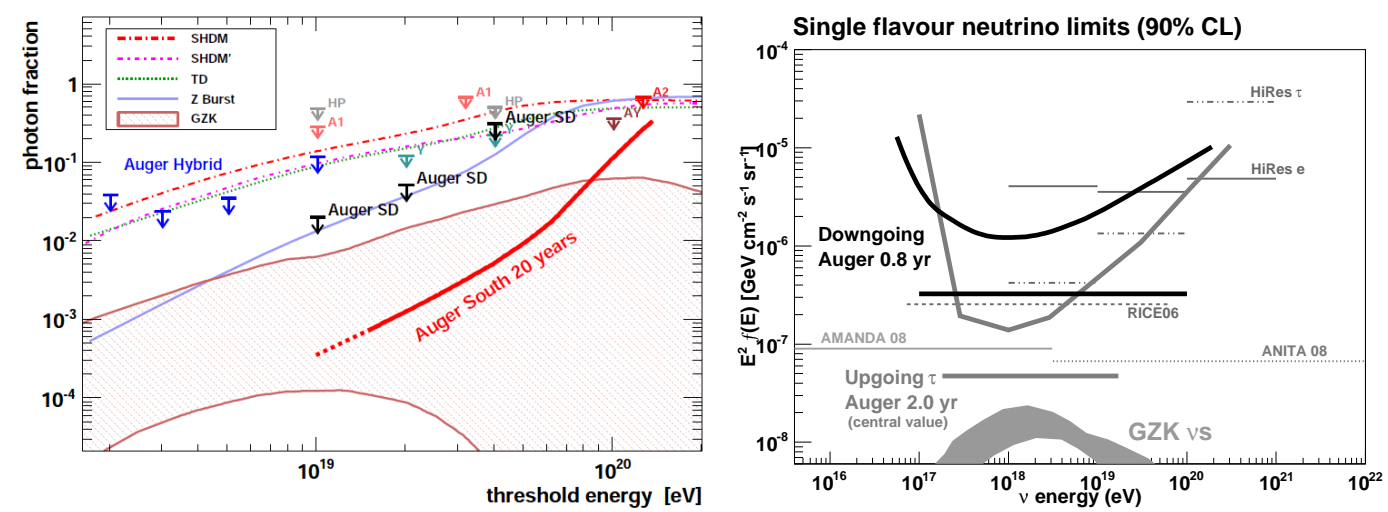

Figure 4: Left panel: Upper limits at 95\% confidence level on the photon fraction of the integral cosmic ray flux for Auger (Hybrid and SD )as a function of the energy. Right panel: Upper limits on the diffuse high-energy neutrino flux in differential and integral format. 